\title{
Implementación de Tic en las prácticas educativas de la educación superior
}

\section{Implementation of Tic in educational practices for higher education}

\author{
Germán Darío Hémbuz Falla1 \\ Edith Polo Osso² \\ Cristian Camilo González Camacho ${ }^{3}$ \\ Universidad SurColombiana
}

\section{RESUMEN}

Desde su llegada las tecnologías de la información y la comunicación (TIC) han simplificado la vida del ser humano de manera

$1 \quad$ Docente Investigador Tiempo Completo Facultad de Economía y Administración de la Universidad Surcolombiana. Cordinador Grupo de Investigación CRE@ Categoría B MINCIENCIAS. Doctorado en Ciencias Sociales, Universidad de Manizales-CINDE. Magister Gerencia del Talento Humano, Universidad de Manizales. Especialista en Alta Gerencia, Universidad Surcolombiana. Especialista Gerencia de Mercado, UCC. Administrador de Empresas Universidad EAN. german.hembuz@usco.edu.co Código https:// orcid.org: 0000-0002-9976-8225 Google Scholar: Hémbuz Falla, Germán Darío - Colombia, Huila - Neiva.

2 Investigadora Universidad Surcolombiana. Programa Administración de Empresas de la Facultad de Economía y Administración de la Universidad Surcolombiana. Grupo de Investigación CRE@ Categoría B MINCIENCIAS. U20152140918@usco.edu.co - Código https://orcid. org: 0000-0003-4691-7212- Tel. +57315 6167436 - Colombia, Huila - Neiva.

3 Investigador Universidad Surcolombiana. Programa Contaduría Pública de la Facultad de Economía y Administración de la Universidad Surcolombiana. Grupo de Investigación CRE@ Categoría B MINCIENCIAS. U20151136192@usco.edu.co - Código https://orcid.org: 0000-0002-3409-0230 - Tel. +57 3178758507 - Colombia, Huila - Neiva. que han sido potencializadas desde el campo de la educación, optimizando los procesos de aprendizaje de los individuos en cada nivel académico. Incluso, hay discursos que han entrado en contradicción, cuestionando la relación entre las prácticas educativas y la implementación de herramientas tecnológicas, que cuestionan la calidad de la educación. En el presente artículo se presenta una revisión teórica focalizada en la implementación de herramientas tecnológicas en las practicas formativas de diversas instituciones de educación superior a nivel nacional e internacional, con el propósito de indagar la manera en que las universidades están ejecutando sus procesos educativos. Concretando, se discute con sentido crítico los logros del profesorado de la Universidad Surcolombiana sobre la ejecución de su quehacer docente, que determina el grado de implementación de las herramientas tecnológicas como estrategia didáctica, teniendo como punto 
de referencia que ahí, en la implementación de las TIC, inicia la anhelada calidad educativa, que hoy ofrece oportunidades de formación de profesionales íntegros e individuos justos para un nuevo sentido de sociedad.

\section{PALABRAS CLAVE}

Educación, Profesores, Estudiantes, Tecnologías, Aprendizaje

\section{ABSTRACT}

Since information and communication technologies (ICT) arrived, have simplified life of human being. In such a way that they have been potentiated from the field of education, optimizing the learning processes of individuals at each academic level. There are even discourses that have come into contradiction, questioning the relationship between educational practices and implementation of technological tools, which question quality of education. This article presents a theoretical review focused on implementation of technological tools for training practices of various higher education institutions at national and international level, with purpose of investigating the way in which universities are executing their educational processes. Specifically, achievements of professors at Surcolombiana University on execution of their teaching work that determines degree of implementation in technological tools as a didactic strategy are discussed with a critical sense, having as a point of reference that there, in implementation of ICT, begins quality education; which today offers training opportunities for upright professionals and fair individuals for a new sense of society.

\section{KEY WORDS}

Education, Professors, Students, Technologies, Learning

\section{INTRODUCCIÓN}

Uno de los aspectos más entrañables que ha ofrecido calidad en las relaciones humanas ha sido la dedicación de tiempo y amor, lo cual se fortalece a través de la comunicación. Es importante mencionar que comunicar ha implicado una presencia física de los agentes, que desde los primeros tiempos de la existencia del ser humano y años más tarde no garantizaba un flujo de comunicación permanente. No obstante, con la irremediable llegada de la modernidad y la posmodernidad esta situación ha cambiado. Los procesos de comunicación a través de los avances tecnológicos y las oportunidades de recortar distancias cada vez más se fortalecen. Por tanto, es válido aseverar que, "la distancia solo es un símbolo de separación, y la comunicación un símbolo de cercanía". (Anyertz, s.f.)

La comunicación elimina la brecha que abre la distancia. Y las tecnologías son su mejor aliado. Por consiguiente, las telecomunicaciones siempre han posibilitado la interacción, más en estos tiempos donde el contacto físico es más limitado, dada la realidad de la pandemia del COVID-19, por lo que el uso de dispositivos, permiten no perder el contacto y las relaciones como alternativas que abren oportunidad de acceso al conocimiento. Hoy han avanzado tanto, que en sus múltiples manifestaciones han hecho realidad el anhelo de ver y hablar al tiempo con quien se encuentra a kilómetros de distancia.

El diccionario de la Real Academia Española define las telecomunicaciones como un "sistema de transmisión y recepción a distancia de señales de diversa naturaleza por medios electromagnéticos" (Real Academía Española, 2021, pág. s.p.); se trata de un conjunto de transferencias, que permiten a los agentes enviar y recibir mensajes de tipo verbal como en una conversación telefónica y no verbal un envió de imágenes y fotografías mediante aplicaciones 
como WhatsApp, Facebook, Instagram, Twiter, entre otros.

Uno de los más destacables efectos con la presencia del COVID -19 ha generado en Colombia y el mundo la obligatoria transformación de las comunicaciones en ambientes físicos por ambientes virtuales. Esto ha llevado a un cambio extremo en el modo de operar de los ciudadanos en cada ámbito de su vida. En efecto, las compras virtuales han sido de carácter casi obligatorio. En consecuencia, los pagos mediante transacciones virtuales y la adquisición de bienes mediante el servicio a domicilio han aumentado. Medidas que han sido adoptadas en pro de mitigar el impacto de este virus en la ciudadanía, para mayor efectividad han atravesado los planos laboral y educativo.

En cuanto al ámbito laboral, las personas empezaron a interactuar con una nueva forma de cumplir con sus labores diarias. Se trata del teletrabajo,

una forma de organización laboral que consiste en el desempeño de actividades remuneradas o prestación de servicios a terceros utilizando como soporte las tecnologías de información y comunicación para el contacto entre el trabajador y la empresa, sin requerirse la presencia física del trabajador en sitio específico de trabajo. (Ministerio de Tecnologías de la Información y la Comunicación , 2016).

Como es de notar, el teletrabajo ha sido una pieza fundamental para la economía de las regiones en tiempos de confinamiento, donde se ha dado un mayor uso de las tecnologías de la información y comunicación como sucede en los servicios de educación. El Doctor Inge Molenaar, argumenta que, la inteligencia artificial es vital en términos educativos; asevera que en estas aplicaciones se halla un estimulante para la innovación educativa y eficacia en el uso de herramientas digitales (UNESCO, 2020).
En este orden de ideas, la relación enseñanza aprendizaje a través de medios virtuales está siendo prometedora, lleva a cuestionar la gigante brecha entre el deber ser de la educación y lo que en realidad es. Sin embargo, las prácticas de algunos docentes $e$ instituciones que enseñan mediante la virtualidad dejan mucho que desear. Su carente dominio tecnológico deja como resultado grandes vacíos en el aprendizaje de sus estudiantes y en la calidad del sistema educativo nacional. Siendo así, el analfabetismo digital es uno de los principales obstáculos en los procesos de formación de los seres humanos, más aún si participan en programas de formación profesional.

El riesgo es perpetuar un significativo número de docentes carentes de habilidades que permitan integrar las herramientas informáticas con estrategias didácticas, porque su formación académica se enmarcó en un contexto en el que las tecnologías no tenían la fuerza de hoy. El tablero de tiza era y acrílico fueron dispositivos muy cercanos a la innovación educativa, mientras no se visualizara la necesidad de enseñar de manera distinta.

Formar mediante entornos virtuales en muchas instituciones educativas del nivel superior se percibe como compromiso exclusivo de los docentes, aunque se evidencia que la institucionalidad aún no ofrece plataformas robustas que fomenten el aprendizaje en línea. El educador debe dotar personalmente los procesos educativos con herramientas tecnológicas de manera autónoma. Esto significa un riesgo educativo dado el desconocimiento de los docentes en materia digital, y más, en el desinterés institucional por capacitarlos. Lo que explica la validez del discurso de Manuel Unigarro Gutiérrez (2020): "Estamos haciendo educación remota [...] utilizamos tecnologías y herramientas virtuales, pero no es educación virtual". 
La desigualdad socioeconómica es otra de las barreras que limitan la labor docente y que se hace ostensible en los entornos virtuales de aprendizaje. Muchos estudiantes carecen de herramientas necesarias para desarrollarse en el actual contexto de la educación. La conexión a internet es una de ellas; "conectar a los 23,8 millones de colombianos que no cuentan con ese beneficio, es uno de los retos más grandes que tiene el país." (Ministerio de Tecnologias de la Información y la Comunicación, 2019)

Por otra parte, los desalentadores resultados educativos obtenidos en el último año son consecuencia de la inequidad social y económica que se manifiesta con el difícil acceso a internet de la mitad de los colombianos, suprimiendo en los docentes cualquier posibilidad de fortalecer sus prácticas educativas. Este es un conflicto al que se enfrentan de dos maneras:

$\checkmark \quad$ Estudiantes que por su pésima conexión no se presentan puntualmente a clase y se ven obligados a no participar en la misma dejando en el educador incertidumbre y preocupación sobre su desempeño académico sin más opción que reprobarlos.

$\checkmark \quad$ Docentes destinados a fracasar con sus estrategias de enseñanza, porque sus estudiantes no cuentan con las herramientas necesarias para ser implementadas.

Un mayor desafío para los educadores se entrevé con aquellos estudiantes que tienen un buen servicio de internet, pero que no están preparados para una educación a distancia y que se habituaron a la presión que ejercía la presencia física de sus profesores. Al apagar la cámara y desactivar el micrófono algunos estudiantes se reservan cumplir con sus deberes académicos, como estar atento a la clase, tomar apuntes, realizar aportes y desarrollar las actividades. No hay un compromiso por parte del estudiante.

Resulta paradójico que siendo "nativos tecnológicos" las generaciones presentes obtienen los mejores resultados con la educación presencial que mediante la virtualidad, discusión que ha permitido el hallazgo de significativos aportes en torno al uso de medios virtuales por parte del profesorado. El analfabetismo digital no es exclusivo de los docentes. Que los estudiantes dominen el uso de herramientas como Tik Tok, Snapchat y Facebook Live entre otras, no significa que estén alfabetizados digitalmente.

Chiecher Costa \& Lorenzati Blengino (2017) mencionan:

No basta con disponer de acceso a tecnologías y ser capaz de operarlas desde una perspectiva tecnológica; también es necesario disponer de conocimientos pedagógicos y didácticos que avalen y den sustento a las propuestas de enseñanza mediadas por TIC (pág.267).

Por tal motivo, es indispensable que los docentes se capaciten en el uso técnico de las herramientas TIC y que los estudiantes se formen en la aplicación de estas en entornos académicos.

Otro importante hallazgo esta relacionado con los instrumentos tecnológicos como principal fuente de entretenimiento para los individuos en la modernidad y se posicionan como figura motivacional. Vera, León \& Bastidas (2017) consideran que "los medios virtuales promueven la construcción y asimilación de conocimientos significativos y perdurables en los estudiantes, mediante un ambiente motivador" (pág. 388).

Capacitar a estudiantes y docentes en las herramientas tecnológicas aplicadas a la educación puede ser una solución viable para muchos de los conflictos que suceden diariamente en el contexto educativo.

\section{METODOLOGÍA}

Para la realización del presente artículo de revisión y el respectivo hallazgo de documentos 
bibliográficos se realizó el proceso de búsqueda en la base de datos Scopus, a través del servicio de suscripción por acceso a docentes y estudiantes de la Universidad Surcolombiana.

Un primer paso consistió en determinar el tema a abordar y sus palabras claves. Para ello, se aplicó una matriz que permitió hacer relaciones entre las palabras claves, sus sinónimos, combinación de términos y su respectiva traducción al inglés. Concluido este ejercicio, se propusieron diferentes ecuaciones booleanas. La primera: ("teaching practice" OR "pedagogical practice") AND (Apply OR implement) AND (Technological tools) AND (Educational space OR classroom) AND (university OR undergraduate level). La segunda: (Teacher* OR Educator) AND (Technologies* OR Technique OR Development OR Advance OR progress* OR innovation* OR Investigation OR Research OR Stady). Y la tercera: Teacher* OR Profess* OR "Literacy workers" OR "Teaching profession" OR Instructor OR educator). Luego, se generó un proceso de filtración en la búsqueda haciendo uso de documentos publicados en los últimos cinco años y como paso final, se procedió a elegir los títulos acordes al tema en estudio y a realizar su respectiva lectura que produce el desarrollo aquí planteado.

\section{DESARROLLO}

El crecimiento económico y social de los países está íntimamente ligado al nivel educativo de sus habitantes, motivo por el que las instituciones educativas se enfrentan al reto de formar íntegramente a sus estudiantes. En virtud de lograr la anhelada metamorfosis en los países que, dé inicio, a cambios reales y necesarios, a acciones justas y equitativas, que logren equilibrar su economía y el alcance del progreso. Gran parte de esta responsabilidad recae en las Instituciones de Educación Superior (IES) dada su función de proveer profesionales que respondan adecuadamente a un mundo destinado al cambio. $Y$ es que en la constante evolución de las sociedades se han transfigurado los modos en que interactúan y aprenden los seres humanos, alineando a los servicios de educación superior para que sean más innovadores en sus procesos de enseñanza con el propósito de alcanzar la calidad educativa.

Hoshimjon, Bakhtiyor y Muzaffar (2020) anuncian que "la fuerza de cualquier país está determinada por su potencial. $Y$ esto depende directamente de la calidad de la educación" (pág. 1.064). Siendo así, se hace obligatorio formar ciudadanos y profesionales íntegros y comprometidos en las instituciones de educación superior. En Colombia, se hace necesario implementar la normatividad expresada en el Sistema de Aseguramiento de la Calidad de la Educación Superior, que luego de un proceso valorativo acredite su calidad como "testimonio que da el Estado sobre la calidad de un programa o institución con base en un proceso previo de evaluación en el cual intervienen la institución, las comunidades académicas y el Consejo Nacional de Acreditación (CNA, 2020).

El proceso de formación académica se ha visto afectado en muchos países principalmente por el cierre de las instituciones educativas. Una estrategia de la UNESCO para mitigar esta situación "apoya la implementación de programas de educación a distancia a gran escala y recomienda aplicaciones y plataformas educativas abiertas que las escuelas y los maestros pueden utilizar para llegar a los alumnos de forma remota" (ONU, 2020).

La innovación educativa es una pieza clave en el alcance de dicha calidad. Especialmente, si se tiene en cuenta que actualmente la educación en Colombia relacionada con el uso de las TICS se ve aminorada en sus cualidades; siendo necesario la implementación de herramientas TIC, que de acuerdo con Strickland (2004, citado en Vinueza y Simbaña, 2017) se definen como "aquellos recursos, herramientas y programas que se utilizan para procesar, administrar $y$ 
compartir la información mediante diversos soportes tecnológicos" (p.358).

En consonancia, Romero, Lopez, Tejada y Garay (2018) mencionan una serie de aspectos positivos en lo que a la implementación de estas herramientas en el ámbito educativo se refiere, destacando las tecnologías de la información y la comunicación que posibilitan el libre acceso a la educación a un mayor número de personas; esta iniciativa en países como Colombia es muy favorable cuando una minoría privilegiada tienen la facilidad económica para sufragar los gastos que la educación presencial implica, como compra de libros, fotocopias, alimentación y transporte, entre otros. Estas tecnologías son un estímulo educativo, ya que permiten un acercamiento entre el aprendizaje y la realidad de quien aprende, lo que explica que las TIC contribuyen a mejorar la práctica docente y la motivación del discente.

Las nuevas generaciones desempeñan roles personales, familiares y sociales, mediante la virtualidad; pero en el contexto educativo se hallan confinados en la enseñanza tradicional, que en nada motiva al aprendizaje. Es en este punto que las TIC representan parte sustancial en los procesos formativos que estimulan al estudiantado. Siendo así, Andersons (citado en Sorayyaei \& Iskandar 2020) plantea que la adecuación en tecnologías permite ajustar los procesos de enseñanza a los intereses de los estudiantes, los cuales están íntimamente ligados a los elementos digitales, estableciendo un aprendizaje significativo.

Las tecnologías transforman las prácticas educativas. Livingstone (2011 citado en Torres, A. Torres, B. y Sousa 2017) afirma que se han convertido en un importante apoyo a la educación tradicional. Para mejorar el aprendizaje de los estudiantes "además de tener acceso a las tecnologías, los profesores deben adoptar la tecnología informada" (Levyn y Tsybulsky 2017, citados en Kolikant, Martinovik y Milner-Bolotín 2020, p.202)

Implementar nuevas tecnologías se ha vuelto un reto para las instituciones de educación superior y en respuesta a esta inevitable exigencia, se hace necesario determinar la capacidad de los docentes universitarios a nivel general, porque en muchas ocasiones se cree que ni el personal docente ni el estudiantado están preparados para el cambio. Ejemplo de ello se evidencia en estudios sobre la capacidad de adaptación de los docentes en lo referente al conocimiento de contenido pedagógico y tecnológico; los resultados aplicados a la Universidad de Sindh llevaron a una misma conclusión: "Si bien esta Universidad no es rica en recursos TIC, los profesores están bien equipados con los conocimientos de uso de los instrumentos tecnológicos y tratan de implementarlos en su proceso de enseñanza-aprendizaje" (Soomro, Bano, Imtiaz, Bhatti, Nazi y Parveen, 2018, pág.208). Pese a la alta capacidad de uso de las herramientas informáticas, hay un vacío en la educación superior del continente asiático que se resume en limitados recursos tecnológicos por parte de las IES. Farley -Riple y Buttram (citados en Sarno 2019) mencionan que "estas herramientas pueden mejorar la capacidad de los profesores que tienen habilidades limitadas en la toma de decisiones basada en datos".

Es esencial, se requiere la adopción por parte del profesorado de las IES de recursos tecnológicos en sus prácticas docentes. Zubieta et al (citado en Orozco, Delgado, Cabezas, Martínez y Solís 2016) menciona que "la frecuencia del uso determina el nivel de aceptación tecnológica de una organización o institución" (p.141), es decir que para conocer el porcentaje de implementación de herramientas TIC es fundamental determinar la constancia con que son aplicados.

Para las instituciones educativas y en particular las del nivel superior es necesario que cuenten 
con las tecnologías del aprendizaje y el conocimiento (TAC), que de acuerdo con Lozano (2011), se enfocan en "conocer y explorar los posibles usos didácticos que las TIC tienen para el aprendizaje y la docencia." (p.46). Es con el apoyo de estas herramientas como se liderarán estrategias institucionales que permitan la innovación educativa.

Por otro lado, se identifican experiencias en la implementación de herramientas TIC en la educación superior, tal como lo señala Vega, Candela y Stendardi (2018) quienes realizaron un estudio sobre Proyectos de Innovación Educativa (PIE) de la Universidad La Laguna en España, como estrategia para fomentar la innovación en educación. Proyectos como "Programa de apoyo a la docencia presencial mediante TIC", el cual busca formar al cuerpo docente en la implementación de aulas virtuales, "Cursos en línea masivos y abiertos" que propician una formación de acceso libre y gratuita, "Materiales Didácticos Digitales" que se fundamenta en la realización anual de una convocatoria, con el fin de crear estrategias que se puedan adaptar y reutilizar en otras instituciones.

Referirse a la implementación de las herramientas tecnológica no es algo sencillo, en Colombia es importante que los educadores desarrollen competencias tecnológicas que fortalezcan su acción docente.

En la última década se ha venido implementando por medio del Ministerio de Educación Nacional MEN-, apoyado en el marco de las políticas de calidad y de equidad de La Revolución Educativa, la formulación de estándares básicos de competencias en tecnología e informática como parte de los requerimientos de la Ley General de Educación (citado en Hernández, Gamboa y Ayala, 2014, p.5).
La Ley 115 del Congreso de la República de Colombia menciona en el artículo 5 numeral 5 como importante "la adquisición y generación de los conocimientos científicos y técnicos más avanzados, humanísticos, históricos, sociales, geográficos y estéticos, mediante la apropiación de hábitos intelectuales adecuados para el desarrollo del saber" (Ley 115, 1994, pág. 2)

Es notable en la normatividad colombiana que enseñar mediante TIC es el primer paso a la educación de calidad. En un $85 \%$ docentes y directivos docentes de distintas universidades de la región norte del país, han coincidido en "considerar que las TIC han contribuido, de forma alta, a mejorar los procesos de comunicación, en los procesos de enseñanza y calidad educativa impartida, así como en los procesos administrativos llevados a cabo en sus instituciones" (Said-Hung 2017, pág. 464).

No obstante, la legislación educativa y la percepción del cuerpo administrativo y docente en mención difieren en gran medida de las prácticas educativas que tienen lugar en las IES. En concreto, Valencia, Said-Hung y Brandle (2017) concluyeron que:

6 de cada 10 docentese ubica en HPTicSuperior, es decir son docentes que llevan a cabo actividades orientadas a analizar, evaluar y crear contenidos y conocimientos bajo la mediación de las TIC. (p.226)

Significa que un $60 \%$ de los docentes encuestados desarrolla habilidades tecnológicas que implementa en su acción docente, mejorando significativamente la calidad de los procesos educativos. Sin embargo, sigue siendo cuantioso el porcentaje de educadores que se enmarcan en una educación tradicional, ausente de estas imprescindibles herramientas.

Con la intención de dar respuesta al interrogante sobre cuál es la implementación 
de las tecnologías que hacen los docentes universitarios en Colombia, Said-Hung, Diaz, Molinares, Barreto, Ballesteros, Vergara y Ordoñez (2015) expresan que:

\begin{abstract}
Suelen hacer un uso medio-alto de recursos aplicados por las mismas instituciones académicas de las que hacen parte (web docente, catálogo de la materia, foros), con un cierto nivel de interactividad asincrónica entre el docente y sus alumnos (por ejemplo, en el caso de los foros) (p.286).
\end{abstract}

Un importante elemento por valorar respecto a cómo están implementando las herramientas TIC los docentes universitarios, es que en los espacios educativos de utilización de herramientas tecnológicas no hay equilibrio en las respuestas de los estudiantes. Jiménez, Martelo y Peña (2017) mencionan "que tanto las universidades colombianas como los organismos gubernamentales realizan inversiones en innovación tecnológica" (pág. 230)

Debido al positivo impacto que las herramientas TIC generan en los procesos educativos, persiste el interés de las universidades por seguir innovando en sus espacios académicos. Romero et al (2018) sostienen que "son diversas las propuestas de innovación que se están realizando en Educación Superior" (s.p).

Es por ello, que en la amplia esfera educativa y como resultado de las múltiples transiciones en las sociedades modernas, han emergido algunos términos que le añaden valor semántico a proyectos innovadores como el de la creación de materiales didácticos digitales. Los recursos educativos digitales abiertos (REDA) es uno de ellos; los que iniciaron bajo un sencillo formato en el que la digitalización estaba ausente (Hodgkinson \& Arinto, 2017).

La Organización de las Naciones Unidas para la Educación, la Ciencia y la Cultura (UNESCO,
2016) lo definieron inicialmente como un conjunto de herramientas de enseñanza y aprendizaje de libre acceso, por ende, de fácil gestión, implementación y reutilización dada su condición de gratuidad como los libros y revistas en las bibliotecas públicas a las que todos pueden acceder sin costo alguno. Una conceptualización que más adelante y debido a los avances tecnológicos especialmente, se le atribuyó el carácter digital. Tal es el caso de los videoclips de YouTube que son elegidos y adaptados a diversas comunidades educativas, una de las múltiples herramientas digitales de libre acceso de las que disponen los educadores. Canva es uno de los REDA más implementados en las universidades, además de su asequibilidad por su versatilidad, brinda herramientas en línea para crear diseños propios. Lo realmente significativo de este tipo de herramientas, es que los usuarios pueden rediseñar y reutilizar sus productos Canva (2020).

Aprender y enseñar en un entorno virtual ha sido un reto, pero con la llegada del COVID 19 la educación tiene la posibilidad de idear estrategias en donde los REDA permitan educar a distancia con medios virtuales de aprendizaje innovadores. Al respecto, Williams (2016) expone que la educación no ha evolucionado de la forma esperada, pese a los constante avances tecnológicos, pues continúan las fallas en la adopción de rutas tecnológicas propias en la educación superior.

Cabe resaltar que en la Universidad Surcolombiana, en efecto, hay una representación del paulatino incremento que ha tenido la implementación de los Recursos Educativos Digitales Abiertos en sus practicas formativas. En la investigación de Hembuz \& Ramos (2015) se evidencia que hasta hace cinco años la aplicación de instrumentos tecnológicos por parte de catedráticos y docentes de planta se limitaba a la presentación de trabajos elaborados mediante office, los 
trabajos escritos se realizaban en Word, la medicion de datos numericos en Excel y las exposiciones eran presentadas mediante Power Point. Una realidad de la que no se difiere mucho actualmente, porque la educación continúa fundamentandose, y con dificultad, en pocas y muy básicas herramientas digitales, cuando el campo tecnológico propone una gran variedad de instrumentos que están enfocadas en los REDA como Flipped Classroom, entre otros, que permiten planteamientos del aula invertida en espacios presenciales, virtuales o blended (combinados). De tal forma,son los estudiantes quienes se preparan para orientar los tópicos de la clase vinculándose directamente con las prácticas pedagógicas.

Desde la Universidad del Internet (UNIR, 2020) se afirma que "este método de enseñanza ha cobrado importancia en los últimos años ante la necesidad de cambiar el sistema tradicional de aprendizaje para adaptarlo a las necesidades actuales [...] Con el Flipped Classroom se aprende haciendo y no memorizando" (s.p)

Valente (2014 citado en Pavanelo \& Lima, 2021) recuerda la importancia del aula invertida como "una modalidad de E-learning en la que el contenido y los instrumentos se estudian en línea antes de que el estudiante asista al aula, lo que ahora se convierte en el lugar para trabajar los contenidos ya estudiados" (pág.742). El estudiante tiene gran dominio de los tópicos a abordar una vez ingresa al salón de clases. Anticipadamente los ha desarrollado y el objeto de asistir al aula es socializar con sus compañeros y profesor lo aprendido, complementando los saberes adquiridos y eliminando dudas. Bergamann \& Sams (citados en Gonçalves \& Quaresma, 2018) mencionan que "la clase gira en torno a los alumnos, no al profesor, él está presente únicamente para proporcionar comentarios de expertos" (pág. 67). Algunos de los elementos más significativos de esta estrategia son para Domínguez, Vega, Espitia,
Sanabria, Corso, Serna \& Osorio (2015) "el fomento del pensamiento crítico [...] promueve el aprendizaje a cargo del propio estudiante, la responsabilidad, la autorregulación, el uso de la tecnología" (p. 514). Algunos desarrollos en el nuevo mundo digital del estudiantado permiten considerar las siguientes estrategias.

- El aula invertida es una importante estrategia pedagógica y didáctica que al alterar el papel docente-estudiante en el aula mediante la implementación de las herramientas tecnológicas, no solo marca la diferencia entre la educación tradicional y la actual, donde el centro del aprendizaje para la primera era el docente. Además, se presenta como una innovadora oportunidad para que sea el estudiante quien edifique su propio conocimiento sin olvidar al docente en su rol de guía en los procesos formativos.

- La innovación educativa fortalece el sistema de educación, allí el estudiante es constructor directo de su propio aprendizaje en donde el e-learning desempeña un papel predominante no solo por su alta capacidad de ser abierto, sino también porque acude al llamado de la educación superior en algunos de sus problemas más usuales: tiempo, espacio, recursos físicos y humanos (Junainah, Khatibi \& Ferdous, 2019, pág.17). En los países en vía de desarrollo algunos individuos se ven obligados a producir ingresos para su subsistencia, lo que resta posibilidades a la hora de continuar con el proceso educativo que exige inversión en dinero y tiempo. Así, con el e-learning se tiene una opción que no debería ser obligatoria; por supuesto aumentarían "las oportunidades de la interacción profesor-alumno y la interacción alumno-alumno a través del aprendizaje en línea" (Nan Yang, 2020, pág. 8).

- El e-learning como herramienta pedagógica del REDA representa un componente clave 
en el camino hacia la calidad educativa del nivel superior, mitiga la distancia existente entre enseñanza de calidad y prácticas educativas reales que se tienen como resultado en un ambiente presencial clases con numerosos estudiantes, limitada infraestructura y pocas herramientas pedagógicas que afectan la calidad del aprendizaje.

- La Realidad Aumentada (RA) es otra propuesta educativa que permite conectar la realidad con la virtualidad. El uso de la RA en la educación muestra un potencial para fomentar y mejorar los métodos de aprendizaje tradicionales (Kurilovas, 2020, pág. 2). Con las interfaces de Realidad Aumentada los estudiantes tienen la posibilidad de aprender mediante la acción, ese es el mayor complemento para una educación que sigue fundamentándose en aprendizajes memorísticos.

\section{DISCUSIÓN}

Para nadie es un secreto que las tecnologías han llegado para simplificar la vida del ser humano, existen desde maquinas automatizadas muy sofisticadas, hasta software que facilitan el manejo de grandes cantidades de información. Muchos de estos softwares son útiles para que los docentes complementen su quehacer pedagógico, programas como Excel simplifican tareas optimizando el tiempo de administración $\mathrm{y}$ el manejo de datos, y programas como Siigo Pyme proporcionan acceso a datos empresariales relevantes, y concretos; como también a información esquemática, minimizando el tiempo en cada procedimiento.

En la actualidad la educación se ha enfrentado a uno de sus más grandes retos, los docentes se han visto obligados a cambiar sus metodologías de enseñanza y los estudiantes han tenido que aprender desde la virtualidad. Ante la acelerada evolución de la tecnología se podría asegurar que esto se veía venir. Algunos docentes han quedado rezagados frente al manejo de las herramientas tecnológicas disponibles en la web y se han visto en la necesidad de capacitarse en el uso de aplicaciones web. Esto produjo un aire de insatisfacción entre los docentes que ejercían la mayor parte de su enseñanza de manera tradicional y los estudiantes manifestaban complicaciones que se podían mitigar con los REDA.

Sin embargo, no dejan de presentarse casos con actores que no cuentan con dispositivos tecnológicos y acceso a internet, y otros quienes aprovechan los beneficios que suministra el campus universitario. También hay casos de educadores que en el momento de implementar las clases virtuales deben enfrentarse al desafío que implica la mala conexión y que afecta directamente el desempeño de la clase, lo cual refleja una distorsión en los mensajes que se anuncian, unido al tardío inicio de las clases por ausencia de un significativo número de estudiantes e incluso del profesor $y$ de estudiantes que no cuentan con las debidas herramientas tecnológicas que les posibilite mostrarse activos en el transcurso de las clases. Este tipo de situaciones alimenta el desinterés de los estudiantes en los procesos de aprendizaje, motivo por el que se muestran ausentes en los espacios de participación. De tal manera desde la Universidad Surcolombiana se formulan nuevos cuestionamientos como:

- ¿Qué procesos tecnológicos motivan el interés de los estudiantes en su formación profesional?

- ¿Qué recursos tecnológicos son necesarios en los diferentes programas académicos para que los estudiantes se apropien de las competencias genéricas y específicas de su profesión?

Con el COVID-19 la Universidad Surcolombiana reemplazó sus aulas fisicas por espacios de 
aprendizaje virtuales como alternancia para la sostenibilidad de sus proyecto académico. Sólo desde el 2020 se evidenció un importante incremento en la adopción de tecnologías en las prácticas pedagógicas apropiadas por el campus universitario. Conviene preguntarse entonces si ¿este nuevo paradigma de educación ha garantizado la calidad educativa? Para lograrlo, se producen interrogantes que invitan a evaluar $¿$ si son suficientes los cambios que ha realizado en el contexto formativo la Universidad Surcolombiana?

Dentro de una extensa gama de aplicaciones que complementan y superan los beneficios de Office como Meet, Zoom , Canva, Genially, Powtoon, Nearpod, Padlet y Airtable entre otras; en la Universidad Surcolombiana ha quedado evidencias que los docentes se han limitado al exclusivo uso de Zoom y Meet para desarrollar los encuentros sincronicos virtuales y Sakai como plataforma de suministro de material didactico y como herramienta valorativa. Pero, ¿Son suficientes estas herramientas para el optimo desarrollo de los procesos formativos en la Universidad surcolombiana? ¿Los estudiantes de la Universidad Surcolombiana tienen la disposición para afrontar los retos que conlleva el uso de herramientas REDA?

Hay una amplia brecha entre lo que se espera lograr y lo que se ha logrado, por tanto, responder a estos interrogantes retan el desarrollo de nuevos proyectos de investigación y desarrollo universitario.

\section{CONCLUSIONES}

Enseñar y aprender a través de herramientas TIC permite optimizar los procesos educativos en un contexto donde el trabajo presencial se complica y más cuando el desarrollo tecnológico no presenta innovaciones significativas en la oferta de programas de las instituciones de educación superior que apenas inician un proceso de adaptación de sus REDA como la
Universidad Surcolombiana; visibilizando los riesgos presentes en el proceso de gestión curricular, mucho más impactado por la pandemia del COVID-19 que llevó al cierre temporal de los campus universitarios para volcarse a nuevas prácticas pedagógicas mediadas por ambientes virtuales de aprendizaje.

Los dispositivos digitales hoy se ven de manera distinta. Son más indispensables, pese a que los sujetos universitarios no sabían darle un uso productivo, y fue así como la implementación de Meet, Zoom y otras plataformas tecnológicas llevaron a ciertas demoras para garantizar espacios de aprendizaje para una educación de alta calidad.

Es importante capacitar a las personas en el uso de las TIC desde un enfoque que posibilite el aprendizaje y el conocimiento, tomando como punto de referencia la multiplicidad de beneficios que en este ámbito ofrecen los recursos educativos digitales abiertos (REDA), que más allá de ofrecer una amplia gama de recursos didácticos que los docentes pueden implementar en sus prácticas educativas y que se ajustan a múltiples necesidades, temáticas y áreas del conocimiento, pueden ser rediseñados y reutilizados en diversos entornos educativos.

Estrategias como el aula invertida que además de complementar los procesos formativos mediante la implementación de tecnologías, le brindan al estudiante un rol activo, permitiéndole ser el constructor de su propio conocimiento.

El e-learning pone fin a los obstáculos generados con relación a su adaptabilidad dado los procesos de obligatorio cumplimiento en tiempos de confinamiento, dándole valor al aprendizaje a distancia y brindando al estudiante libertad en ritmo y tiempo; junto a la realidad aumentada como estrategia que viabiliza la aplicación de instrumentos tecnológicos y la fusión entre realidad y el mundo virtual, como adopción de ese aprendizaje que inicia en la acción. 
Hacer uso de estos recursos tecnológicos en el plano educativo dejará en evidencia que hay otras maneras de accionar la relación enseñanza aprendizaje, logrando que los docentes pierdan el temor que por mucho tiempo ha sido generado a causa de su carente dominio tecnológico; motivando a los estudiantes en el camino que conduce al aprendizaje y entre todos, haciendo posible el ideal de una educación de calidad aún en tiempos de confinamiento.

\section{REFERENCIAS BIBLIOGRÁFICAS}

Anyertz. (s.f.). Literato . Obtenido de https:// www.literato.es/p/MzEyNTA/

Canva. (2020). Canva. Obtenido de https://www. canva.com/es_co/educacion/

Chiecher Costa, A. C., \& Lorenzati Blengino, K. P. (2017). Estudiantes y tecnologías. Una visión desde la 'lente' de docentes universitarios. RIED. Revista Iberoamericana de Educación a Distancia, vol. 20, núm. 1, 2017, pp. 261-282.

CNA. (2020). Sistema Nacional de Acreditación en Colombia. Bogotá D.C.: CNA.

Domínguez, L. C., Vega, N. V., Espitia, E. L., Sanabria, Á. E., Corso, C., Serna, A. M., \& Osorio, C. (2015). Impacto de la estrategia de aula invertida en el ambiente de aprendizaje en cirugía: una comparación con la clase magistral. Revista Biomédica

Gonçalves Pereira, Z. T., \& Quaresma da Silva, D. (2018). Metodología activa: Flipped Classroom y sus prácticas en Educacion basica. REICE. Revista Iberoamericana de Calidad, Eficacia y Cambio en Educación.
Hémbuz Falla, G. D., \& Ramos Polanía, A. (2015). Prácticas de Innovación en la Formación de Profesionales en la Facultad de Ciencias Económicas y Administrativas de la Universidad Cothuila y Surcolombiana que contribuyen a Fortalecer su Politica de Calidad Académica. Grupo de Investigación GIAC, Universidad Cohuila, Neiva, Colombia.

Hernandez Suarez, C. A., Gamboa Suarez, A. A., \& Ayala García, E. T. (2014). COMPETENCIAS TIC PARA LOS DOCENTES DE EDUCACION SUPERIOR. Congreso Iberoamericano de Ciencia, Tecnología, Innovación y Educación.

Hodgkinson Williams, C., \& Arinto, P. (2017). Adopción e Impacto de REA en el Sur Global. African Minds, Centro Internacional de Investigación para el Desarrollo e Investigación sobre Recursos Educativos Abiertos.

HoshimjonBunatovich, U., BakhtiyorKhidayevich, D., \& Muzaffar Abdurakhmonovich, O. (2020). La Importancia De Los Métodos De Enseñanza Innovadores Modernos En El Más Alto Sistema De Educación De Uzbekistán. Revista de revisiones críticas.

Jiménez Pitre, I., Martelo Gómez, R. J., \& Peña Pertuz, M. (2017). Diagnóstico sobre Accesibilidad e Integración Digital dentro del Sector Universitario Colombiano. SABER, CIENCIA Y Libertad ISSN: 1794-7154 Vol. 12, No.1 Enero-Junio 2017 Págs. 225-235.

Junainah Abd Hamid, A. K. (2019). La adecuación deUTAUT-3en Interpretación académica y adopción del e-Learning en entornos educativos. Escuela de Graduados 
en Administración, Administración y Ciencias de la Universidad, Shah Alam, Malasia.

Kolikant, David; Martinovic, D., \& Milner-Bolotin, M. (2020). Profesores y Enseñanza de STEM en la Era Digital. (c) Springer Nature Suiza AG 2020.

Kurilovas, E. (2020). Sobre la toma de decisiones basada en datos para una educación de calidad. Elsevier Science \& Technology.

Lozano, R. (2011). De las TIC a las TAC: Tecnologías del Aprendizaje al Conocimiento. Anuario ThinkEPI, 2011, v. 5.

Ministerio de Educación Nacional. (1994). Ley 115. Bogotá.

Ministerio de Tecnologías de la Información y la Comunicación . (13 de julio de 2016). Obtenido de https://www.youtube.com/ watch?v=PqLJpiVAzKk

Ministerio de Tecnologias de la Información y la Comunicación. (19 de mayo de 2019). Obtenido de https://www.mintic.gov.co/ portal/inicio/Sala-de-Prensa/MinTICen-los-Medios/100837:La-mitad-deColombia-no-tiene-internet

Nam, Y. (2020). Elearning para una Enseñanza de Calidad en Educación Superior. (C) Springer Nature Singapore Pte Ltd.

ONU. (5 de marzo de 2020). Noticias ONU. Obtenido de https://news.un.org/es/ story/2020/03/1470641

Orozco Cazco, G. H., Cabezas González, M., Martínez Abad, F., Delgado Altamirano, J. E., \& Solís Mazón, M. E. (2016). Factores determinantes en la aceptación de las TIC por la Universidad profesores en su práctica docente.
Cuarta Conferencia Internacional sobre Ecosistemas Tecnológicos para Potenciar la Multiculturalidad - TEEM'16.

Pavanelo, E., \& Lima, R. (2021). Aula invertida: el análisis de una experiencia en la disciplina. Boletin de Educación Matemática - BOLEMA.

Romero Andonegui, A., Tejada Garitano, E., López de la Serna, A., \& Garay Ruiz, U. (2018). La Innovación Tecnológica mediada por las TIC en Educación Superior. En Experiencias pedagógicas e innovación educativa Aportaciones desde la praxis docente e investigadora. España.

Real Academía Española. (2021). Diccionario de la Real Academia Española. Madrid: R.A.E.

Said-Hung, E., Díaz-Granados, F. I., Jabba Molinares, D., Ricardo Barreto, C., Ballesteros, B., Vergara, E., \& Ordoñez, M. (2015). FORTALECIMIENTO PEDAGÓGICO EN LAS UNIVERSIDADES EN COLOMBIA A TRAVÉS DE LAS TIC. CASO REGIÓN CARIBE. Educación XX1, 18(2), 277304, doi:10.5944/educXX1.14605.

Said-Hung, E., Valencia-Cobos,, J., \& González Prieto, E. (2017). La promoción de experiencias de innovación educativa en TIC en los centros escolares. Caso región Caribe colombiana. Estudios Pedagógicos XLIII, N 1: 457-473, 2017.

Sarno Owens, J., McLennan, J., L. Hustus , C., Haines-Saah , R., Mitchell , S., Clifton S, M., \& Troutman , A. (2019). Aprovechamiento de la tecnología para facilitar el uso por parte de los maestros de una intervención específica en el aula: Evaluación de la boleta de calificaciones diaria. Sistema en línea (DRC.O). ( ) 
Springer Science + Business Media, LLC, parte de Springer Nature 2019.

Soomro Saira, B. S. (2018). Adaptación TPACK entre profesores de departamentos de educación y TIC en la Universidad de Sindh, Pakistán. Revista Internacional de Aplicaciones y Ciencias Informaticas Avanzadas.

Sorayyaei Azar, Ali N. H. (2020). La aplicación de tecnologías TIC (aprendizaje de idiomas asistido por dispositivos móviles, gamificación y realidad virtual) en la enseñanza de inglés dispositivos móviles, gamificación y realidad virtual) en la enseñanza de inglés en Malasia ... Revista Universal de Investigación Educativa.

Torres, M. F., Sousa , A. J., \& Torres , R. T. (2017). Replanificación pedagógica y tecnológica: un exitoso estudio de caso sobre integración y habilidades transversales para estudiantes de primer año de ingeniería. Springer Science + Business Media Dordrecht .

UNESCO. (2016). Recursos Educativos Abiertos. Obtenido de https://es.unesco. org/themes/tic-educacion/rea

UNESCO. (21 de Julio de 2020). Organización de las Naciones Unidas para la Educación, la Ciencia y la Cultura. Obtenido de https://es.unesco.org/news/que-ayudapueden-proporcionar-tecnologiasinteligentes-durante-pandemia

Unigarro Gutiérrez, M. (17 de abril de 2020). Evaluar por competencias en la educación superior. Bogotá: Universidad Cooperativa de Colombia. Obtenido de https://www.youtube.com/ watch?v=o_0QKUjmEB0
UNIR. (2020 de Marzo de 2020). UNIR. Obtenido de https://www.unir.net/educacion/ revista/flipped-classroom/

Valencia-Cobos, J., Brändle Señan , G., \& Said-Hung, E. (2017). NIVEL DE APROVECHAMIENTO DOCENTE DE LAS TIC EN COLOMBIA. Revista del Curriculum y Formación del Profesorado.

Vega Navarro, A., Candela Sanjuán, B. A., \& Stendardi, D. (2018). Infraestructura e iniciativas institucionales para impulsar la innovación educativa en la universidad. International Journal of Information Systems and Software Engineering for Big Companies (IJISEBC).

Vera-Mora, G., León-Acurio, J., \& Bastidas Zambrano, L. (2017). El papel de los medios virtuales en la enseñanza de la educación secundaria del siglo XXI. Revista Cientifica Dominio de las Ciencias.

Vinueza Vinueza , S. F., \& Simbaña Gallardo , V. P. (2017). Impacto de las TIC en la Educación Superior en el Ecuador. Revista Publicando.

Williams, R. (2 de Octubre de 2016). Youtube. Obtenido de https://www.youtube.com/ watch?v=muhAbVkicEw\&t=6s 